\title{
Abstract \\ The spectrum of Rheumatoid Arthritis. A single unit experience
}

\author{
Tennakoon TMIS $^{1 *}$, Nissanka TM ${ }^{1}$, Bandaranayake BMVC ${ }^{2}$ \\ ${ }^{1}$ Teaching Hospital, Kurunegala, Sri Lanka \\ ${ }^{2}$ Teaching Hospital, Kandy, Sri Lanka
}

\begin{abstract}
Background

Rheumatoid arthritis (RA) is a chronic inflammatory disease affecting the synovium, leading to joint damage and bone destruction causing severe disability and increased mortality. Studies on the spectrum and expression of RA in Asian population are limited in the literature. Objective of the study was to describe the demographic, clinical and laboratory characteristics of RA patients followed up at Rheumatology Department, Teaching Hospital, Kurunegala. Method

Descriptive study of both first visit and follow up patients diagnosed with RA was conducted during 3 week period starting from 20th July 2014. The relevant data were recorded and analyzed after excluding incomplete data.

\section{Results}

Of the 89 patients recruited, $14(16 \%)$ were males and $75(84 \%)$ were females. The mean age of patients was 53.05 $(+/-16.8)$ years and the mean disease duration at presentation was 11.6 months. The upper limb joints were affected more than the lower limbs and the most commonly involved joint was the proximal interphalangeal (PIP) joint $(85.4 \%)$ followed by the wrist joint $(80.9 \%)$, the knee $(78.7 \%)$, metacarpophalangeal (MCP) joints $(76.4 \%)$, ankle joint (65.2\%), elbow joint (49.4\%), shoulder joint (49.4\%) and small joints of foot (37.1\%). The least affected joints were the hip (9\%) and the temperomandibular (TM) joint (4.5\%). Only $40.4 \%$ were seropositive. The mean ESR at presentation was $73.4(+/-31.33)$ while mean Heamoglobin was $10.8 \mathrm{mg} / \mathrm{dl}(+/-1.38)$ and platelet count was 336000 (+/-96.68).
\end{abstract}

\section{Conclusions}

Demographic and clinical characteristics were similar to world statistics except lower rate of seropositivity and higher female predominance.

Key words: Rheumatoid Arthritis; Demographic data; Clinical Characteristics

Copyright: (C) 2015 Tennakoon TMIS et al. This is an open access article distributed under the Creative Commons Attribution License, which permits unrestricted use, distribution, and reproduction in any medium, provided the original work is properly cited.

* Correspondence : Indika_st@yahoo.com

Cite this abstract as: Tennakoon TMIS, Nissanka TM, Bandaranayake BMVC. The spectrum of Rheumatoid Arthritis. A single unit experience. Anuradhapura Medical Journal 2015;9 (2Supp):S38.

DOI: http://dx.doi.org/10.4038/amj.v9i2Supp.7587 
